\title{
Patient-Reported Outcomes and Aesthetic Results after Immediate Breast Reconstruction Using Human Acellular Dermal Matrices: Results of a Multicenter, Prospective, Observational NOGGO-AWOGyn Study
}

\author{
Jens-Uwe Blohmer ${ }^{\mathrm{a}} \mathrm{b}, \mathrm{h}$ Lea Beier ${ }^{\mathrm{a}, \mathrm{h}} \quad$ Andree Faridi $^{\mathrm{b}, \mathrm{c}}$ Christine Ankel $^{\mathrm{d}}$ \\ Barbara Krause-Bergmann ${ }^{\mathrm{e}}$ Stefan Paepke ${ }^{\mathrm{b}, \mathrm{f}, \mathrm{h}}$ Christine Mau ${ }^{\mathrm{g}, \mathrm{h}}$ \\ Maren Keller ${ }^{h}$ Hans Joachim Strittmatter ${ }^{b, h}, i$ Maria Margarete Karsten ${ }^{a}$

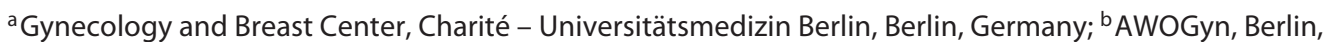

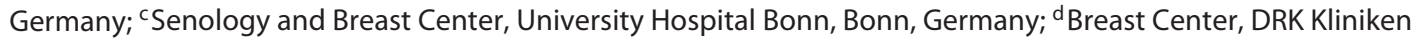

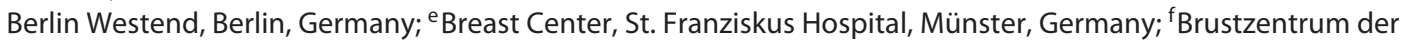 \\ Technischen Universität München Klinikum Rechts der Isar Comprehensive Cancer Center, Munich, Germany;

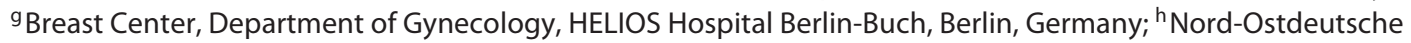 \\ Gesellschaft für Gynäkologische Onkologie e.V., Berlin, Germany; 'Department of Gynecology, Rems-Murr-Hospital \\ Winnenden, Winnenden, Germany
}

\section{Keywords}

Patient-reported outcomes - Acellular dermal matrix . Breast reconstruction - Quality of life - Aesthetic result

\begin{abstract}
Background: With the increased use of acellular dermal matrices (ADMs) in implant-based breast reconstructions (IBBRs), the evaluation of patient-reported outcomes becomes more important. Methods: Patients who underwent an immediate human ADM-assisted, submuscular IBBR were included in this noninterventional, multicenter, prospective cohort study. Patients with primary reconstruction (cohort A) and patients with a revision surgery after capsular fibrosis (cohort B) were followed up for 12 months after surgery. Quality of life (EORTC BR-23) and patient and surgeon satisfaction scores (1 ["very satisfied"] to 6 ["not satisfied"]) with the outcome and the aesthetic result evaluated by 2 independent, external experts were assessed. Results: Eightyfour patients were enrolled in the study. The mean patient
\end{abstract}

karger@karger.com www.karger.com/brc

Karger $\frac{1}{\%}$

BOPEN ACCESS
(C) 2020 The Author(s)

Published by S. Karger AG, Basel

This is an Open Access article licensed under the Creative Commons Attribution-NonCommercial-4.0 International License (CC BY-NC) (http://www.karger.com/Services/OpenAccessLicense), applicable to the online version of the article only. Usage and distribution for commercial purposes requires written permission. satisfaction score was $2.1 \pm 0.8$, with higher satisfaction in cohort B $(p=0.041)$. The score did not change significantly during the follow-up $(p=0.479)$. The mean satisfaction score of the surgeons was $2.0 \pm 0.7$; it was also higher in cohort $B$ $(p=0.016)$ and showed no changes over time $(p=0.473)$. The mean aesthetic result was $2.2 \pm 0.7 .92 .9 \%$ of the patients completed at least 1 quality of life questionnaire. Body image and sexual functioning increased during follow-up. One year after surgery, the mean scores were $77.2 \pm 22.5$ and 44.7 \pm 27.3 , respectively. Conclusion: The level of satisfaction among patients and surgeons and the score of the aesthetic result were constantly high among patients after ADM-assisted IBBR. Higher satisfaction scores could be observed after revision surgery caused by capsular fibrosis (cohort B) compared to primary reconstruction (cohort A). Quality of life increased during the first year after surgery.

(c) 2020 The Author(s)

Published by S. Karger AG, Basel

Jens-Uwe Blohmer and Lea Beier contributed equally to this article. 


\section{Introduction}

Breast reconstructions after mastectomies in breast cancer patients have shown to improve patient-reported outcomes when compared to patients without reconstruction following mastectomy $[1,2]$. Breast reconstructions improve the patient's quality of life and self-esteem and reduce the emotional and cognitive burden of disease [2]. Implant-based reconstructions positively affect the physical condition and functional status of breast cancer patients [2]. In traditional subpectoral implant-based reconstructions, the muscle tissue coverage of the implant can often only be achieved for the upper and lower medial quadrants, while the coverage of the lower part of the implant often remains insufficient [3]. This can lead to postoperative discomfort, such as increased implant perception, lack of support, and skin erosion [3]. Since their introduction in breast surgery in 2001, acellular dermal matrices (ADMs) evolved to a cornerstone in implantbased breast reconstruction [4]. The use of an ADM enables complete coverage of the implant, thus stabilizing the implant and improving control of the breast fold [3, 5-7]. However, the advantages of ADM-assisted breast reconstruction, including improved aesthetic outcome [8-10] and reduced risks of capsular fibrosis $[6,11,12]$, come with an increased risk of certain complications, such as seroma, skin necrosis, infections, and loss of implant [12-14].

Most studies evaluating the use of ADMs in breast reconstruction focus on clinical outcomes, without assessing patient-reported outcomes [15]. From a patient's perspective, an improved quality of life and satisfaction with the surgical result are key outcomes of the breast reconstruction [16]. The aim of our study was not only to evaluate complication rates, which have been reported previously [17], but also to prospectively assess patients' quality of life, patient and surgeon satisfaction, and the aesthetic outcome in patients undergoing an immediate human ADM-assisted breast reconstruction after skinand nipple-sparing mastectomy.

\section{Materials and Methods}

\section{Study Design}

We conducted a noninterventional, multicenter, prospective cohort study in 11 sites with experience in performing ADMassisted breast reconstructions in Germany (German Clinical Trials Register ID DRKS00007587). Ethical approval was obtained from the ethics committees of all participating sites. Women undergoing a human ADM-assisted immediate submuscular implant-based breast reconstruction after skin- and nipple-sparing mastectomy were enrolled in this study. Patients were followed up 1 day, 1 week, 1, 3, 6, and 12 months after surgery. Patient characteristics were collected prior to surgery. During follow-up, occurrence of complications, patient satisfaction, surgeon satisfaction, and quality of life (from 1 week after surgery onwards) were assessed and photographs were taken. Details on complication rates in the present study have been reported previously [17].

Patient and surgeon satisfaction were assessed on a scale from 1 ("very satisfied") to 6 ("not satisfied"). Aesthetic results were assessed by 2 external experts, who were experienced breast surgeons not involved in enrollment, using standardized photographs (front and side perspective) at 1 week, 1, 3, 6, and 12 months after surgery on a scale from 1 ("very satisfied") to 6 ("not satisfied"). No further criteria for the evaluation of the aesthetic outcome by the experts were defined. The experts were blinded in terms of study cohort and did not receive any information on the patient characteristics, including occurrence of complications.

Quality of life was assessed using the EORTC BR-23 questionnaire. BR-23 is a validated and standardized questionnaire for measuring the quality of life in breast cancer patients.

\section{Study Population}

Women who underwent an ADM-assisted immediate breast reconstruction after skin- and nipple-sparing mastectomy using the human ADM Epiflex ${ }^{\circledR}$ (Deutsches Institut für Zell- und Gewebeersatz [DIZG] gGmbH, Berlin, Germany), $\geq 18$ years of age, and providing written informed consent were eligible for inclusion in the study. Physicians were asked to consecutively enroll all eligible patients.

Patients were enrolled into 2 cohorts: cohort A - patients who received a primary breast reconstruction, and cohort $\mathrm{B}$ - patients undergoing a revision surgery after capsular fibrosis.

Patients with an autoimmune disease, known contraindication against mesh-assisted or plastic reconstructive breast surgery, and previous radiotherapy (only cohort A) and pregnant or breastfeeding women were excluded from this study.

\section{Statistical Analysis}

Patients who matched all inclusion criteria and none of the exclusion criteria were included in the analyses. Descriptive analysis was performed providing absolute frequencies and proportions for categorical variables, as well as means and standard deviations for continuous variables. Quality of life was analyzed as stated in the EORTC QLQ-C30 scoring manual [18]. All subscales of the EORTC BR-23 questionnaire have a range from 0 to 100 points. For the functional scales, body image and sexual function, which were evaluated in the present study, a higher score represents a better function and, therefore, higher quality of life.

Changes in the mean patient and surgeon satisfaction scores and the aesthetic scores during the follow-up visits were analyzed using the Friedman test. Differences in the mean satisfaction and quality of life scores between the study cohorts were analyzed using the $t$ test or Mann-Whitney $U$ test as appropriate. The impact of the variables age (continuous variable), smoking status, diagnosis of histologically confirmed breast cancer, precancerosis, adjuvant chemotherapy, side of reconstruction (unilateral vs. bilateral), follow-up (continuous), postoperative complications (including loss of implant, seroma, rash, Baker grade III/IV capsular fibrosis, and infection), and type of surgery (primary vs. revision surgery after capsular fibrosis) on patient satisfaction was analyzed using univariate and multivariate linear regression analyses.

No hypotheses were predefined for these exploratory analyses. A two-sided $p$ value of $<0.05$ was considered statistically significant. All statistical analyses were performed using IBM SPSS version 25 (IBM, USA). 
Table 1. Study population characteristics

\begin{tabular}{lccc}
\hline Patient characteristics & $\begin{array}{l}\text { Total } \\
(n=84)\end{array}$ & $\begin{array}{l}\text { Cohort A } \\
(n=53)\end{array}$ & $\begin{array}{l}\text { Cohort B } \\
(n=31)\end{array}$ \\
\hline Bilateral reconstruction, $n(\%)$ & $28(33.3)$ & $20(37.7)$ & $8(25.8)$ \\
Age in years, mean \pm SD & $44.5 \pm 11.9$ & $41.4 \pm 9.9$ & $49.8 \pm 13.2$ \\
Smokers, $n$ (\%) & $18(21.4)$ & $9(17.0)$ & $9(29.0)$ \\
BMI, kg/m ${ }^{2}$, mean \pm SD & $22.1 \pm 2.8$ & $22.0 \pm 3.0$ & $22.2 \pm 2.5$ \\
Follow-up in monthss, mean \pm SD & $9.7 \pm 3.9$ & $9.6 \pm 3.9$ & $9.8 \pm 4.0)$ \\
Breast cancer, $n(\%)$ & $48(57.1)$ & $34(64.2)$ & $14(45.2)$ \\
Precancerosis/DCIS, $n(\%)$ & $16(19.0)$ & $10(18.9)$ & $6(19.4)$ \\
Freedom from pain, $n(\%)$ & $77(91.7)$ & $49(92.5)$ & $28(90.3)$ \\
Adjuvant chemotherapy, $n(\%)$ & $9(10.7)$ & $6(11.3)$ & $3(9.7)$ \\
Adjuvant endocrine therapy, $n(\%)$ & $33(39.3)$ & $27(50.9)$ & $6(19.4)$ \\
NSAID use, $n$ (\%) & $67(79.8)$ & $42(79.2)$ & $25(80.6)$ \\
Complications* & $30(35.7)$ & $18(34.0)$ & $12(38.7)$ \\
\hline
\end{tabular}

* Complications include loss of implant, any seroma, infection, rash and Baker grade III/IV capsular fibrosis. BMI, body mass index; DCIS, ductal carcinoma in situ; NSAID, non-steroidal anti-inflammatory drug.

not satisfied 6

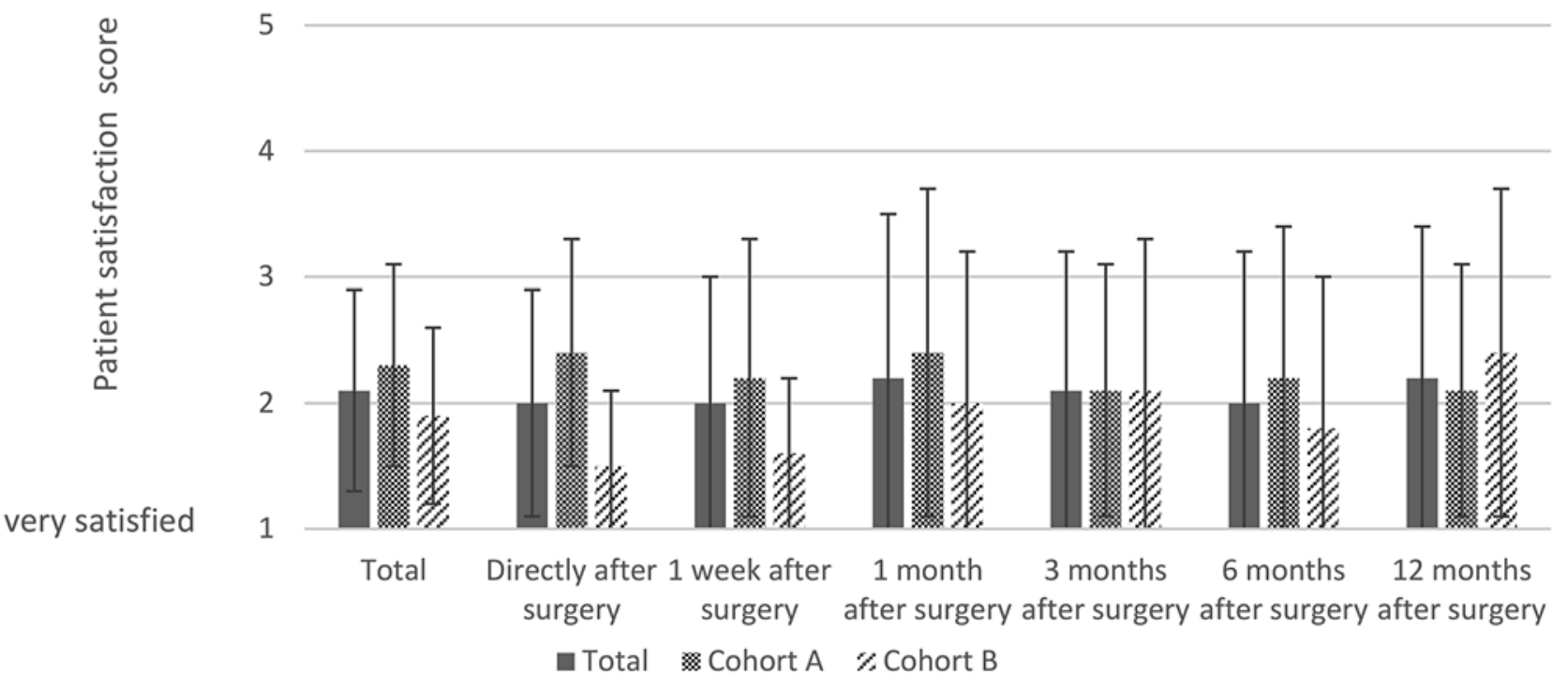

Fig. 1. Average patient satisfaction with the surgical outcome.

\section{Results}

\section{Patient Population}

Between January 2015 and September 2018, 84 eligible patients were included in the study. Fifty-three underwent primary reconstruction (cohort A) and 31 underwent a revision surgery after capsular fibrosis (cohort B). Twentyeight women received bilateral reconstruction, leading to 112 reconstructions in total. Characteristics of the study population are reported in Table 1. Patients were followed up for $9.7 \pm 3.9$ months on average. The mean age was 44.5 \pm 11.9 years. $21.4 \%$ of the patients were smokers. The mean body mass index was $22.1 \pm 2.8 \mathrm{~kg} / \mathrm{m}^{2} .57 .1 \%$ had histologically confirmed invasive breast cancer, and in 19.0\%, a precancerosis like ductal carcinoma in situ was diagnosed. During follow-up, $91.7 \%$ of the patients reported to be free of pain during movement. $79.8 \%$ of the patients were taking nonsteroidal anti-inflammatory drugs (NSAID) at some point during the follow-up. One year after surgery, $7.8 \%$ of the patients reported to take NSAIDs. $10.7 \%$ of the 


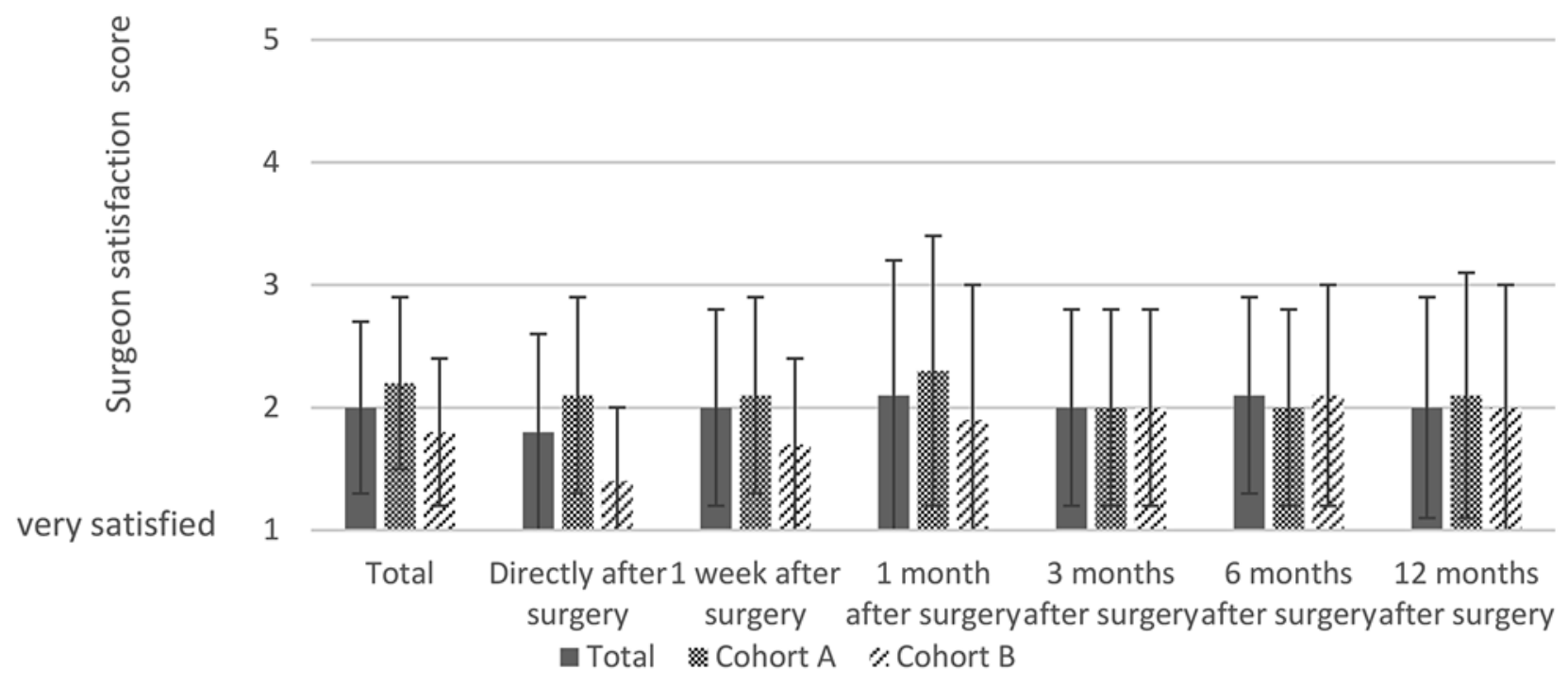

Fig. 2. Average surgeon satisfaction with the surgical outcome.

patients received an adjuvant chemotherapy, 39.3\% received postoperative endocrine therapy. During follow-up, $35.7 \%$ of all patients experienced a postoperative complication, such as loss of implant, seroma, infection, rash, and Baker grade III/IV capsular fibrosis.

\section{Patient Satisfaction}

Data on patient satisfaction for at least 1 study visit were available for 83 patients (cohort A: 52 patients; cohort B: 31 patients). The mean patient satisfaction scores over the study period are shown in Figure 1. The average self-reported patient satisfaction over the complete study period was $2.1 \pm 0.8$. The patient satisfaction did not change significantly during follow-up $(p=0.479)$. However, for the study visits 6 months and 1 year after surgery, data were only available for 41 patients (cohort A: 25 patients; cohort B: 16 patients) and 43 patients (cohort A: 25 patients; cohort B: 18 patients), respectively. The overall patient satisfaction was better in cohort B than in cohort A $(1.9 \pm 0.7$ and $2.3 \pm 0.8$, respectively; $p=0.041)$.

\section{Factors Influencing Patient Satisfaction}

Results of the univariate and multivariate analyses are reported in online supplementary Table 1 (see www. karger.com/doi/10.1159/000509568 for all online suppl. material). In the multivariate analysis, no factors influencing the satisfaction of patients could be identified. In the univariate analysis, longer follow-up $(p=0.015)$ and revision surgery after capsular fibrosis (vs. primary reconstruction, $p=0.033$ ) were significantly associated with higher satisfaction. Patients with complications had a significantly shorter follow-up time than patients without complications $(8.5 \pm 4.4$ and $10.4 \pm 3.5$ months, respectively; $p=0.029$ ).

\section{Surgeon Satisfaction}

Data regarding the surgeon satisfaction with the surgical outcome for at least 1 study visit was available for all patients. The average surgeon satisfaction scores during follow-up are shown in Figure 2. The overall average satisfaction score of the surgeons was $2.0 \pm 0.7$ and did not change statistically significantly over time $(p=0.473)$. However, as for the patient satisfaction, data for the study visits 6 and 12 months after surgery were only available for less than half of the patients (41 patients; cohort A: 25 patients; cohort B: 16 patients) at both visits. The overall surgeon satisfaction was better in cohort $B$ than in cohort A $(1.8 \pm 0.6$ and $2.2 \pm 0.7$, respectively; $p=0.016)$.

\section{External Experts}

The average aesthetic scores are shown in Figure 3. For 78 patients, at least 1 postoperative photography was available. The average ranking of the external experts was $2.2 \pm 0.7$. In cohort A, the mean aesthetic score was $2.3 \pm$ 
5

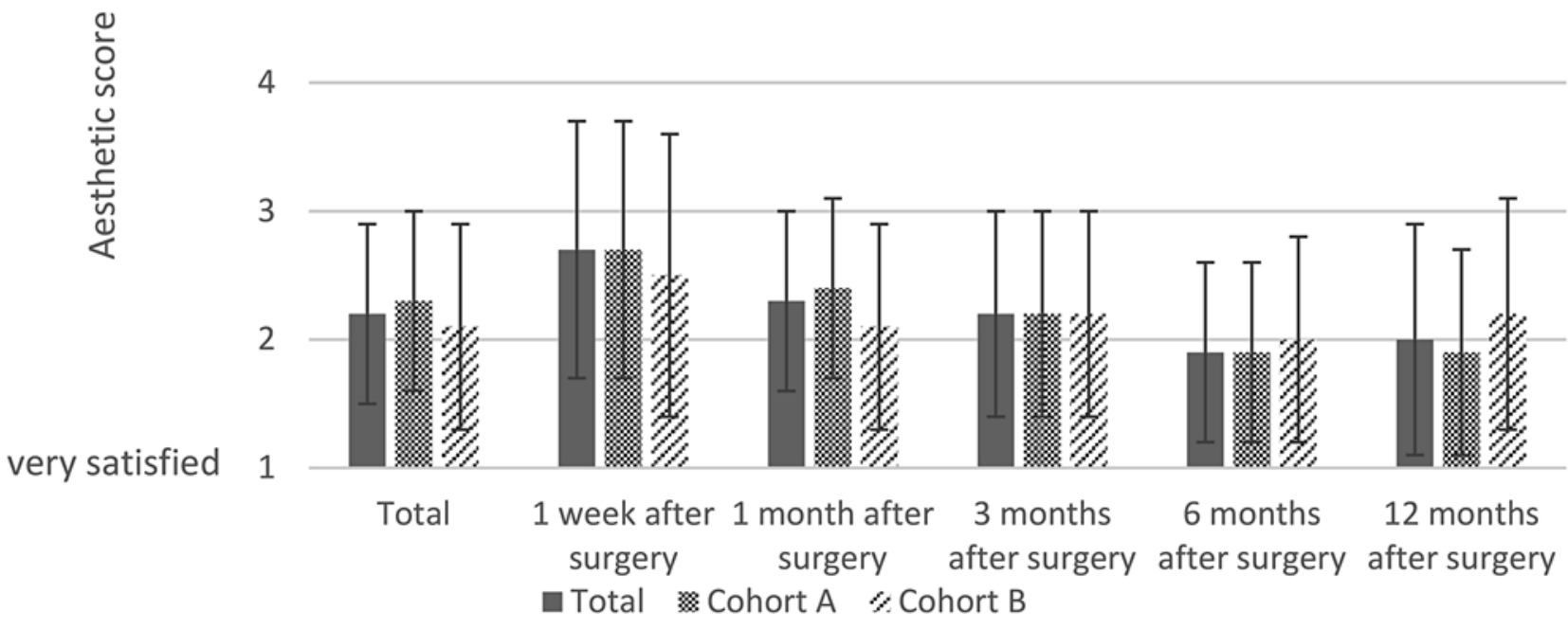

Fig. 3. Average aesthetic scores judged by 2 independent external experts.

Table 2. Average quality of life score

\begin{tabular}{|c|c|c|c|c|c|c|c|}
\hline \multirow{2}{*}{$\frac{\text { BR-23 }(\text { mean } \pm S D)}{\text { Body Image }}$} & \multicolumn{2}{|l|}{ Total } & \multicolumn{2}{|c|}{ Cohort A } & \multicolumn{2}{|c|}{ Cohort B } & \multirow{2}{*}{$\frac{p \text { value }}{0.113}$} \\
\hline & $n=78$ & $69.8 \pm 22.8$ & $n=50$ & $66.8 \pm 22.2$ & $n=28$ & $75.3 \pm 23.3$ & \\
\hline Sexual functioning & $n=75$ & $39.9 \pm 25.2$ & $n=50$ & $40.2 \pm 23.2$ & $n=25$ & $39.1 \pm 29.1$ & 0.854 \\
\hline
\end{tabular}

0.7 , and in cohort $\mathrm{B}$, it was $2.1 \pm 0.8$, with no significant differences between the groups $(p=0.242)$. The mean aesthetic score differed significantly between the study visits $(p=0.015)$.

\section{Quality of Life}

92.9\% of the patients completed at least 1 quality of life questionnaire. Results of the average quality of life scores are reported in Table 2 . Results per study visit are reported in online supplementary Table 2.

No significant differences in the average scores for body image and sexual functioning were present between the cohorts. The mean score of the questionnaire domain "body image" was $69.8 \pm 22.8$. During follow-up, the mean body image score significantly increased $(p=0.000)$, with a mean score of $77.2 \pm 22.5$ twelve months after surgery.

Sexual functioning was ranked $39.9 \pm 25.2$ on average. During follow-up, the mean score significantly changed $(p=0.007)$, reaching the highest score at the study visit 6 months after surgery $(48.5 \pm 29.3)$. Twelve months after surgery, the score slightly decreased $(44.7 \pm 27.3)$.

\section{Discussion}

With the increased use of ADMs in breast reconstructions, the evaluation of patient-reported outcomes becomes more important. From a patient's perspective, improved quality of life and satisfaction with the surgical result are key outcomes of the breast reconstruction [16]. In our study, in addition to the previously reported complication rates [17], we prospectively assessed patient-reported outcomes, including patient satisfaction and quality of life, in patients who underwent an ADM-assisted immediate breast reconstruction after skin- and nipplesparing mastectomy with or without prior breast reconstruction. In addition, surgeon satisfaction with the outcome and the aesthetic result judged by 2 independent external experts were assessed. 
Overall, patients and surgeons reported high levels of satisfaction with the outcome showing an average satisfaction score of 2.1 and 2.0, respectively. Patients who underwent a revision surgery after capsular fibrosis (cohort $B$ ) were more satisfied than those after a primary reconstruction (cohort A). The increased satisfaction of patients who received a revision surgery after capsular fibrosis (cohort B) might be partly explained by the negative experience with the primary reconstruction. The overall quality of life did not differ between both cohorts.

In the present study, occurrence of postoperative complications was not significantly associated with decreased patient satisfaction, as reported previously [19]. However, we found the occurrence of complications to be associated with the follow-up time: patients with an uncomplicated postoperative course had a significantly longer follow-up than patients suffering from complications $(p=0.029)$. A longer follow-up, in turn, was found to be associated with increased patient satisfaction.

The relatively high overall complication rates might be explained by the high proportion of patients with confirmed breast cancer and our outcome definition for the study. The most common complication was seroma, as all seromas detected by the routinely conducted ultrasound examinations were noted, including those that did not require any clinical intervention. The association of complications and follow-up time possibly reflects the impact of more severe complications since affected patients might be more likely to be lost to follow-up earlier than patients with less severe complications (e.g., rash, clinically nonrelevant seroma). Thus, the late assessments of patient-reported outcomes might be biased towards greater satisfaction, as more patients without complications contributed to the scores. On the other hand, the average satisfaction and quality of life scores might be reduced due to the many patients responding at the early visits. In general, patient-reported outcomes need some months to return to preoperative levels after surgery. Weichman et al. [20] reported that patients did not fully recover until 3 months postoperatively. $\mathrm{Vu}$ et al. [21] reported that, compared to baseline, patient-reported outcomes decreased 2 months after surgery and returned to preoperative levels only by 6 months after surgery.

Postoperative radiotherapy was reported to negatively affect the patients' satisfaction with the outcome [21]. However, since we did not systematically assess this in our study, we are not able to make any statements on it. Similar to radiotherapy, a negative impact on patient satisfaction could also be shown for unilateral reconstructions [19]. Regarding this issue, no connection could be found in our study.

The use of ADMs improves the aesthetic results of breast reconstructions compared to reconstructions without ADMs $[8,10,22]$. In a study by Nguyen et al. [8], the ADM-assisted breast reconstruction scored higher in most of the assessed aesthetic criteria, including breast mound volume, placement, contour, and definition of the inframammary fold. Only scarring was scored higher in the non-ADM group, without reaching significance [8]. Forsberg et al. [10] evaluated the aesthetic outcome based on 5 aesthetic features, including natural contour, symmetry of shape, symmetry of size, position on chest wall, and overall aesthetic appearance, with significantly increased scores for all criteria in ADM-assisted compared to conventional submuscular reconstructions. Direct comparisons with our findings are not feasible, which is due to differing scoring systems. However, in our study, the external experts also ranked the aesthetic outcome good with a mean of $2.2 \pm 0.7$ and no significant differences between the study cohorts. The changes in the aesthetic scores during follow-up should be interpreted with caution, as photographs were not available for every visit in many cases. Thus, the visits consisted of different subpopulations.

The contribution of a woman's satisfaction with her breasts to her psychosocial and sexual well-being has been reported previously [19]. In a cross-sectional study comparing the quality of life in a total of 612 primary breast cancer patients using the EORTC BR-23, the authors found body image to be scored highest in patients who underwent breast-conserving therapy $(83.9 \pm 21.3)$, followed by the scores for postmastectomy autologous $(81.9 \pm 21.0)$ and prosthesis-based reconstructions (77.3 \pm 25.1 ) [9]. The body image score was lowest in patients with mastectomy only $(75.7 \pm 26.0)$. Patients who received chemotherapy up to 6 months prior to enrollment were excluded from that study, and the quality of life was assessed over a median time of 6.3 years postoperatively [9]. In our study, body image was scored $69.8 \pm 22.8$ on average with a significant increase during the first year after surgery. At the latest assessment, the mean score was $77.2 \pm 22.5$. Lagendijk et al. [23] found patients after implant-based reconstruction to have the highest scores on the questionnaire domain "sexual functioning" (mean of $36.6 \pm 24.0$ ). We even found higher rates for sexual functioning in our cohort with an average of $39.9 \pm 25.2$ during the study period and a maximum mean score of 44.7 \pm 27.3 one year after surgery.

However, the interpretation of the EORTC BR-23 questionnaire is limited as it was actually developed to assess the quality of life in breast cancer patients focusing on nonsurgical treatment issues [24]. In the present study, only $57.1 \%$ of the women were diagnosed with breast cancer, and quality of life was only assessed postoperatively. This is why it was not possible to evaluate the pre- and postoperative differences in this regard. A questionnaire commonly used to assess patient-reported outcomes in 
breast surgery is the BREAST-Q. It includes domains like satisfaction with breasts, overall outcome, and process of care, as well as psychosocial, physical, and sexual wellbeing $[25,26]$. A comparison of our study with previous publications using the BREAST-Q questionnaire is therefore restricted.

The data regarding the impact of different surgical techniques on patient-reported outcomes in breast reconstruction are heterogeneous since most of the studies on ADM-assisted surgery focus on clinical outcomes only [15]. It has been shown that patient-reported outcomes after autologous reconstructions are superior to those after implant-based reconstructions $[27,28]$, but the former also have an increased risk of complications [28]. Moreover, previous studies could not find any differences in patient-reported outcomes between breast reconstructions with and without ADMs [15, 29].

Our study is limited by a relatively small sample size and short follow-up period. Thus, the results should be interpreted with caution and be confirmed by further studies with lager sample sizes and a longer follow-up period.

\section{Conclusion}

The awareness regarding the importance of evaluating patient satisfaction and quality of life is increasing in clinical practice. The levels of patient and surgeon satisfaction were constantly high after human ADM-assisted implant-based breast reconstruction following skinand nipple-sparing mastectomy. Patients who received revision surgery due to capsular fibrosis (cohort B) reported higher satisfaction scores than patients with primary reconstruction (cohort A). Furthermore, the scores of the aesthetic results also achieved high levels and quality of life significantly increased during the first year after surgery. Nevertheless, due to the negative impact of complications on patient-reported outcomes, surgeons should carefully decide on which patients they consider suitable for ADM-assisted implant-based breast reconstruction.

\section{Acknowledgement}

The authors acknowledge all study centers for their support during the conduct of the study and all study participants for contributing to this study.

\section{Statement of Ethics}

The study was approved by the ethics committees at all participating sites (ethics committees of the Charité - Universitätsmedizin Berlin, Landesärtzekammer Baden-Württemberg, Otto-von Guericke-Universität an der Medizinischen Fakultät und am Universitätsklinikum Magdeburg, Technische Universität München, Medizinische Hochschule Hannover, Ärztekammer Westfalen-Lippe). Written informed consent was obtained from all study participants in order to participate in the study.

\section{Conflict of Interest Statement}

Andree Faridi is a consultant of pfm Köln and received speaking fees from pfm Köln and DIZG Berlin. Stefan Paepke received fees from pfm, TapMed, Novus Scientific, and DIZG. Christine Ankel is a consultant of pfm Köln. All other authors have no conflicts of interest to declare.

\section{Funding Sources}

The study was unrestrictedly funded by Berliner Krebsgesellschaft e.V., Berlin, Germany; NOGGO e.V., Berlin, Germany; AWOGyn e.V., Berlin, Germany; Förderverein Berliner Brustzentren e.V., Berlin, Germany, and DIZG, Berlin, Germany. The financial sponsors had no role in the data collection and data analysis or the manuscript. The sponsor of the study was NOGGO e.V., Berlin, Germany.

\section{Author Contributions}

Jens-Uwe Blohmer and Lea Beier wrote the manuscript with input from all authors. Lea Beier performed the data analyses. Andree Faridi, Maren Keller, and Jens-Uwe Blohmer contributed to the study design and writing of the study protocol. Jens-Uwe Blohmer, Andree Faridi, Chistine Ankel, Barbara Krause-Bergmann, Stefan Paepke, Christine Mau, Hans Joachim Strittmatter, and Maria Margarete Karsten contributed to data collection and data interpretation.

\section{References}

1 Cordova LZ, Hunter-Smith DJ, Rozen WM. Patient reported outcome measures (PROMs) following mastectomy with breast reconstruction or without reconstruction: a systematic review. Gland Surg. 2019 Aug;8(4): 441-51.

2 Strittmatter HJ, Neises M, Blecken SR. Kriterien der Lebensqualität nach rekonstruktiven Mammakarzinomoperationen. Zentralbl Gynäkol. 2006 Aug;128(4):217-23.

3 Dieterich M, Faridi A. Biological Matrices and Synthetic Meshes Used in Implant-based
Breast Reconstruction - a Review of Products Available in Germany. Geburtshilfe Frauenheilkd. 2013 Nov;73(11):1100-6.

4 Margulies IG, Salzberg CA. The use of acellular dermal matrix in breast reconstruction: evolution of techniques over 2 decades. Gland Surg. 2019 Feb;8(1):3-10.

5 Smith JM, Broyles JM, Guo Y, Tuffaha SH, Mathes D, Sacks JM. Human acellular dermis increases surgical site infection and overall complication profile when compared with submuscular breast reconstruction: an updat- ed meta-analysis incorporating new products. J Plast Reconstr Aesthet Surg. 2018 Nov; 71(11):1547-56.

6 Lee KT, Mun GH. Updated Evidence of Acellular Dermal Matrix Use for Implant-Based Breast Reconstruction: A Meta-analysis. Ann Surg Oncol. 2016 Feb;23(2):600-10.

7 Boháč M, Danišovič L, Koller J, Dragúňová J, Varga I. What happens to an acellular dermal matrix after implantation in the human body? A histological and electron microscopic study. Eur J Histochem. 2018 Jan;62(1):2873. 
8 Nguyen KT, Mioton LM, Smetona JT, Seth AK, Kim JY. Esthetic Outcomes of ADM-Assisted Expander-Implant Breast Reconstruction. Eplasty. 2012;12:e58.

9 Lagendijk M, van Egdom LS, van Veen FE, Vos EL, Mureau MA, van Leeuwen N, et al. Patient-Reported Outcome Measures May Add Value in Breast Cancer Surgery. Ann Surg Oncol. 2018 Nov;25(12):3563-71.

10 Forsberg CG, Kelly DA, Wood BC, Mastrangelo SL, DeFranzo AJ, Thompson JT, et al. Aesthetic outcomes of acellular dermal matrix in tissue expander/implant-based breast reconstruction. Ann Plast Surg. 2014; 72(6):S116-20.

11 Salzberg CA, Ashikari AY, Berry C, Hunsicker LM. Acellular Dermal Matrix-Assisted Direct-to-Implant Breast Reconstruction and Capsular Contracture: A 13-Year Experience. Plast Reconstr Surg. 2016 Aug;138(2):329-37.

12 Ho G, Nguyen TJ, Shahabi A, Hwang BH, Chan LS, Wong AK. A systematic review and meta-analysis of complications associated with acellular dermal matrix-assisted breast reconstruction. Ann Plast Surg. 2012 Apr; 68(4):346-56.

13 Chun YS, Verma K, Rosen H, Lipsitz S, Morris $\mathrm{D}$, Kenney $\mathrm{P}$, et al. Implant-based breast reconstruction using acellular dermal matrix and the risk of postoperative complications. Plast Reconstr Surg. 2010 Feb;125(2):429-36.

14 Hallberg H, Rafnsdottir S, Selvaggi G, Strandell A, Samuelsson O, Stadig I, et al. Benefits and risks with acellular dermal matrix (ADM) and mesh support in immediate breast reconstruction: a systematic review and meta-analysis. J Plast Surg Hand Surg. 2018 Jun;52(3):130-47.

15 Sorkin M, Qi J, Kim HM, Hamill JB, Kozlow JH, Pusic AL, et al. Acellular Dermal Matrix in Immediate Expander/Implant Breast Reconstruction: A Multicenter Assessment of Risks and Benefits. Plast Reconstr Surg. 2017 Dec;140(6):1091-100.
16 Voineskos SH, Nelson JA, Klassen AF, Pusic AL. Measuring Patient-Reported Outcomes: Key Metrics in Reconstructive Surgery. Annu Rev Med. 2018 Jan;69(1):467-79.

17 Beier L, Faridi A, Neumann C, Paepke S, Mau C, Keller M, et al. Human acellular dermal matrix in immediate implant-based breast reconstruction after skin and nipple sparing mastectomy and treatment of capsular fibrosis - results of a multicenter, prospective, observational NOGGO-AWOGyn study. Submitted.

18 Fayers PM, Aaronson NK, Bjordal K, Groenvold M, Curran D, Bottomley A; EORTC Quality of Life Group. The EORTC QLQ-C30 Scoring Manual (3rd edition). Brussels: European Organisation for Research and Treatment of Cancer; 2001.

19 Negenborn VL, Dikmans RE, Bouman MB, Wilschut JA, Mullender MG, Salzberg CA. Patient-reported Outcomes after ADM-assisted Implant-based Breast Reconstruction: A Cross-sectional Study. Plast Reconstr Surg Glob Open. 2018 Feb;6(2):e1654.

20 Weichman KE, Hamill JB, Kim HM, Chen X, Wilkins EG, Pusic AL. Understanding the recovery phase of breast reconstructions: patient-reported outcomes correlated to the type and timing of reconstruction. J Plast Reconstr Aesthet Surg. 2015 Oct;68(10):13708.

21 Vu MM, De Oliveira GS Jr, Mayer KE, Blough JT, Kim JY. A Prospective Study Assessing Complication Rates and Patient-Reported Outcomes in Breast Reconstructions Using a Novel, Deep Dermal Human Acellular Dermal Matrix. Plast Reconstr Surg Glob Open. 2016 Jan;3(12):e585.

22 Vardanian AJ, Clayton JL, Roostaeian J, Shirvanian V, Da Lio A, Lipa JE, et al. Comparison of implant-based immediate breast reconstruction with and without acellular dermal matrix. Plast Reconstr Surg. 2011 Nov;128(5):403e-10e.
23 Lagendijk M, van Egdom LS, van Veen FE, Vos EL, Mureau MA, van Leeuwen N, et al. Patient-Reported Outcome Measures May Add Value in Breast Cancer Surgery. Ann Surg Oncol. 2018 Nov;25(12):3563-71.

24 Chen CM, Cano SJ, Klassen AF, King T, McCarthy C, Cordeiro PG, et al. Measuring quality of life in oncologic breast surgery: a systematic review of patient-reported outcome measures. Breast J. 2010 Nov-Dec;16(6):58797.

25 Pusic AL, Klassen AF, Scott AM, Klok JA, Cordeiro PG, Cano SJ. Development of a new patient-reported outcome measure for breast surgery: the BREAST-Q. Plast Reconstr Surg. 2009 Aug; 124(2):345-53.

26 Cano SJ, Klassen AF, Scott AM, Cordeiro PG, Pusic AL. The BREAST-Q: further validation in independent clinical samples. Plast Reconstr Surg. 2012 Feb;129(2):293-302.

27 Pusic AL, Matros E, Fine N, Buchel E, Gordillo GM, Hamill JB, et al. Patient-Reported Outcomes 1 Year After Immediate Breast Reconstruction: Results of the Mastectomy Reconstruction Outcomes Consortium Study. J Clin Oncol. 2017 Aug;35(22):2499-506.

28 Taylor EM, Wilkins EG, Pusic AL, Qi J, Kim HM, Hamill JB, et al. Impact of Unilateral versus Bilateral Breast Reconstruction on Procedure Choices and Outcomes. Plast Reconstr Surg. 2019 Jun;143(6):1159e-68e.

29 Negenborn VL, Young-Afat DA, Dikmans RE, Smit JM, Winters HA, Don Griot JP, et al. Quality of life and patient satisfaction after one-stage implant-based breast reconstruction with an acellular dermal matrix versus two-stage breast reconstruction (BRIOS): primary outcome of a randomised, controlled trial. Lancet Oncol. 2018 Sep;19(9):1205-14. 